\title{
Acute Exacerbation of COPD Triggered by Pneumonia, and Secondary Spontaneous Pneumothorax (SSP)
}

\author{
Sourya Acharya ${ }^{1}$, Amol Andhale ${ }^{2}$, Yash Gupte ${ }^{3}$, Vidyashree Hulkoti ${ }^{4}$, Akhilesh Annadatha ${ }^{5}$
}

\begin{abstract}
${ }^{1}$ Department of Medicine, Datta Meghe Institute of Medical Sciences (Deemed to be University), Sawangi Meghe, Wardha, Maharashtra, India. ${ }^{2}$ Department of Medicine, Datta Meghe Institute of Medical Sciences (Deemed to be University), Sawangi Meghe, Wardha, Maharashtra, India. ${ }^{3}$ Department of Medicine, Datta Meghe Institute of Medical Sciences (Deemed to be University), Sawangi Meghe, Wardha, Maharashtra, India. ${ }^{4}$ Department of Medicine, Datta Meghe Institute of Medical Sciences (Deemed to be University), Sawangi Meghe, Wardha, Maharashtra, India. ${ }^{5}$ Department of Medicine, Datta Meghe Institute of Medical Sciences (Deemed to be University), Sawangi Meghe, Wardha, Maharashtra, India.
\end{abstract}

\section{INTRODUCTION}

Secondary Spontaneous Pneumothorax (SSP) can rarely complicate Chronic Obstructive Pulmonary Disease (COPD). Infections are common triggers for exacerbations of COPD. COPD with acute exacerbation presents with increasing dyspnoea. We present a rare case of a 75 year old female, who was a known case of COPD and developed right middle lobe pneumonia with partial collapse of the middle lobe along with a secondary spontaneous pneumothorax.

\section{PRESENTATION OF CASE}

A 75-year-old female, a known case of COPD since 20 years presented with fever, cough with expectoration and exaggerated dyspnoea of 5 days duration. Her COPD was well controlled with formoterol $(6 \mathrm{mcg})$ and budesonide $(200 \mathrm{mcg})$ one puff twice a day and oral Doxophylline $400 \mathrm{mg}$ once a day. But her symptoms were not relived. The expectoration was yellowish green in colour. There was no history of haemoptysis, PND, chest pain, and oedema feet.

On examination: Pulse- 118/min, regular, BP- $110 / 76 \mathrm{~mm}$ of $\mathrm{Hg}$, respiratory rate26 cycles / min, accessory muscles of respiration were in action. $\mathrm{SpO}_{2}$ in ambient air was $91 \%$. JVP was normal.

Respiratory system examination revealed absent air entry in right supra clavicular, and mammary areas. Tubular bronchial breath sounds in right axillary area. Cardiovascular and per abdomen examination was normal.

CBC- Hb-14 gm\%, TLC- 14,600/MM 3, with 82\% neutrophilic predominance, KFT, LFT were normal. Blood culture did not reveal any growth. Sputum AFB was negative. HRCT thorax revealed; Partial collapse of right middle lobe with consolidation with right pneumothorax. (Figure 1)

\author{
Corresponding Author: \\ Dr. Sourya Acharya, \\ Professor, \\ Department of Medicine, \\ Datta Meghe Institute of Medical Sciences, \\ Deemed to be University, Sawangi Meghe, \\ Wardha, Maharashtra, India. \\ E-mail: souryaacharya74@gmail.com
}

\section{DOI: $10.14260 / j e m d s / 2020 / 501$}

How to Cite This Article:

Acharya S, Andhale A, gupte $Y$, et al. Acute exacerbation of COPD triggered by pneumonia, and secondary spontaneous pneumothorax (SSP). J. Evolution Med. Dent. Sci. 2020;9(32):2314-2315, DOI: $10.14260 / \mathrm{jemds} / 2020 / 501$

Submission 16-04-2020,

Peer Review 01-07-2020,

Acceptance 07-07-2020,

Published 10-08-2020.

Copyright (C) 2020 JEMDS. This is an open access article distributed under Creative Commons Attribution License [Attribution 4.0 International (CC BY 4.0)] 


\section{Jemds.com}

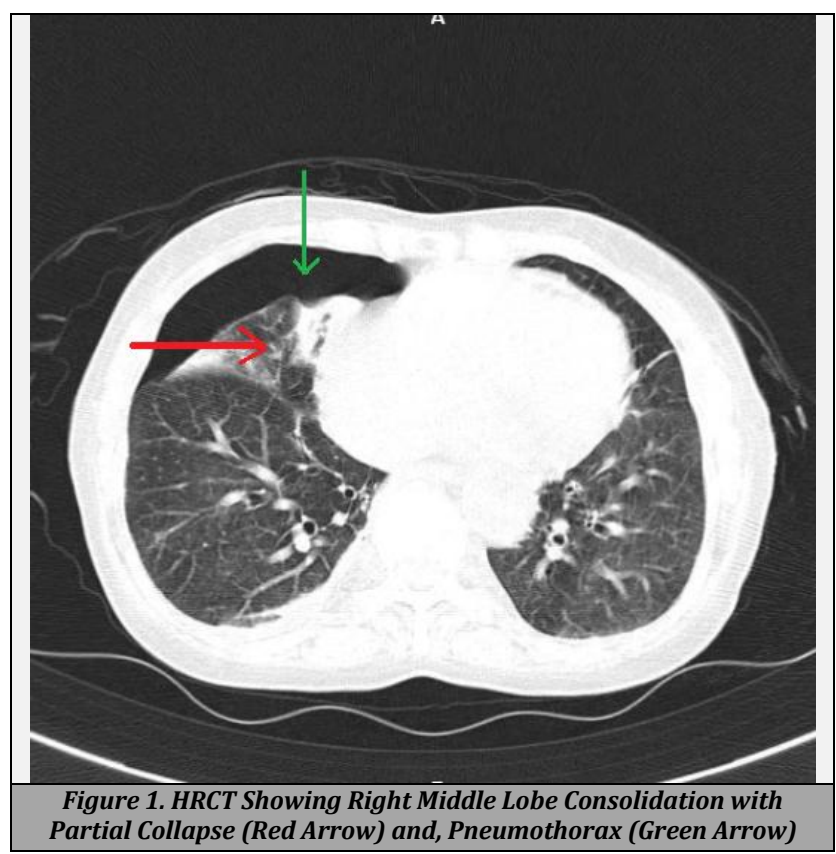

\section{DISCUSSION OF MANAGEMENT}

The patient was treated with supplemental high flow oxygen and tube thoracostomy along with antibiotics and nebulisations with beta 2 agonists and anti-cholinergic.

\section{DISCUSSION}

Acute exacerbations of COPD is conveniently treated with beta agonist and anticholinergic nebulisations, antibiotics, oxygen supplementation, NIPPV, and steroids. Rarely SSP can complicate COPD. Our patient had consolidation/ partial collapse of the middle lobe along with closed pneumothorax that further aggravated the dyspnoea.

\section{Case Report}

A pneumothorax is an accumulation of air in the pleural cavity. Spontaneous pneumothorax (SP) is a cardiopulmonary emergency.[1] SSP usually complicates COPD in females more than 60 years of age. It requires immediate treatment. ${ }^{[2]}$

The most common pathology of SSP in COPD is rupture of an apical sub pleural bleb or bulla which occurs in $50-70 \%$ of cases.[3] Thirty percent of the SSP complicating COPD may not require intercostal tube insertion. ${ }^{[4]}$

Cases of COPD with acute exacerbations who do not improve predictably with adequate therapy, SSP should be ruled out. A second CXR or HRCT might be required.

In this case the crisis might have been triggered by dual phenomena, that is; infection and SSP. SSP occurs more in emphysema predominant COPD. If the pneumothorax occupies less than $15 \%$ of the hemithorax and the patient is asymptomatic then conservative therapy with high flow oxygen is need or else in symptomatic cases the standard treatment is tube thoracostomy.[5]

Financial or Other Competing Interests: None.

\section{REFERENCES}

[1] Choi WI. Pneumothorax. Tuberc Respir Dis (Seoul) 2014;76(3):99-104.

[2] Noppen M, De Keukeleire T. Pneumothorax. Ann Thorac Surg 1993;55(2):372-76.

[3] Chen CH, Liao WC, Liu YH, et al. Secondary spontaneous pneumothorax: which associated conditions benefit from pigtail catheter treatment? Am J Emerg Med 2012;30(1):45-50.

[4] Guo Y, Xie C, Rodriguez RM, et al. Factors related to recurrence of spontaneous pneumothorax. Respirology 2005;10(3):378-84.

[5] Light RW. Management of spontaneous pneumothorax. Am Rev Respir Dis 1993;148(1):245-48. 